\title{
Evolution of genomic sequence inhomogeneity at mid-range scales
} Ashwin Prakash ${ }^{\dagger 1,7}$, Samuel S Shepard ${ }^{\dagger 1,7}$, Jie He ${ }^{2}$, Benjamin Hart ${ }^{3}$,
Miao Chen ${ }^{3}$, Surya P Amarachintha ${ }^{4}$, Olga Mileyeva-Biebesheimer
Jason Bechtel $^{6}$ and Alexei Fedorov*6,7

Address: ${ }^{1}$ Program in Cardiovascular \& Metabolic Diseases Track, Biomedical Sciences, University of Toledo Health Science Campus, Toledo, OH 43614, USA, 2 University of Toledo, Department of Biology, Toledo, Ohio, USA, ${ }^{3}$ Dept of Medical Microbiology \& Immunology, Infection, Immunity \& Transplantation Track, University of Toledo, Health Science Campus, Toledo, OH 43614, USA, ${ }^{4}$ Department of Biological Sciences, Bowling Green State University, Bowling Green, OH - 43403, USA, ${ }^{5}$ Department of Civil Engineering, University of Toledo, Toledo, Ohio, USA, ${ }^{6}$ Program in Bioinformatics and Proteomics/Genomics, University of Toledo Health Science Campus, Toledo, OH 43614, USA and ${ }^{7}$ Department of Medicine, University of Toledo, Health Science Campus, Toledo, Ohio, USA

Email: Ashwin Prakash - ashwin.prakash@utoledo.edu; Samuel S Shepard - samuel.shepard@utoledo.edu; Jie He - jie.he@utoledo.edu; Benjamin Hart - benjamin.hart@utoledo.edu; Miao Chen - miao.chen@utoledo.edu; Surya P Amarachintha - suryaa@bgsu.edu; Olga MileyevaBiebesheimer - omileyn@UTNet.UToledo.Edu; Jason Bechtel - jasonmbechtel@gmail.com; Alexei Fedorov* - alexei.fedorov@utoledo.edu

* Corresponding author †Equal contributors

Published: 5 November 2009

BMC Genomics 2009, 10:5/3 doi:10.1186/|47|-2/64-10-513
Received: 18 March 2009

Accepted: 5 November 2009

This article is available from: http://www.biomedcentral.com/I47I-2/64/10/5/3

(C) 2009 Prakash et al; licensee BioMed Central Ltd.

This is an Open Access article distributed under the terms of the Creative Commons Attribution License (http://creativecommons.org/licenses/by/2.0), which permits unrestricted use, distribution, and reproduction in any medium, provided the original work is properly cited.

\begin{abstract}
Background: Mid-range inhomogeneity or MRI is the significant enrichment of particular nucleotides in genomic sequences extending from 30 up to several thousands of nucleotides. The best-known manifestation of MRI is $C_{P G}$ islands representing CG-rich regions. Recently it was demonstrated that MRI could be observed not only for $G+C$ content but also for all other nucleotide pairings (e.g. $A+G$ and $G+T$ ) as well as for individual bases. Various types of MRI regions are 4-20 times enriched in mammalian genomes compared to their occurrences in random models.

Results: This paper explores how different types of mutations change MRI regions. Human, chimpanzee and Macaca mulatta genomes were aligned to study the projected effects of substitutions and indels on human sequence evolution within both MRI regions and control regions of average nucleotide composition. Over 18.8 million fixed point substitutions, 3.9 million SNPs, and indels spanning $6.9 \mathrm{Mb}$ were procured and evaluated in human. They include I.8 Mb substitutions and I.9 Mb indels within MRI regions. Ancestral and mutant (derived) alleles for substitutions have been determined. Substitutions were grouped according to their fixation within human populations: fixed substitutions (from the human-chimp-macaca alignment), major SNPs ( $>80 \%$ mutant allele frequency within humans), medium SNPs (20\% - $80 \%$ mutant allele frequency), minor SNPs (3\% - 20\%), and rare SNPs (<3\%). Data on short (<3 bp) and medium-length ( $3-50$ bp) insertions and deletions within MRI regions and appropriate control regions were analyzed for the effect of indels on the expansion or diminution of such regions as well as on changing nucleotide composition.

Conclusion: MRI regions have comparable levels of de novo mutations to the control genomic sequences with average base composition. De novo substitutions rapidly erode MRI regions, bringing their nucleotide composition toward genome-average levels. However, those substitutions that favor the maintenance of MRI properties have a higher chance to spread through the entire population. Indels have a clear tendency to maintain MRI features yet they have a smaller impact than substitutions. All in all, the observed fixation bias for mutations helps to preserve MRI regions during evolution.
\end{abstract}




\section{Background}

The protein coding sequences of humans and of most other mammals represent less than $2 \%$ of their genomes. The remaining $98 \%$ is made up of 5 '- and 3 '-untranslated regions of mRNAs $(<2 \%)$, introns $(\sim 37 \%)$, and intergenic regions $(\sim 60 \%)$ [1]. These vast non-protein coding genomic areas, previously frequently referred to as "junk" DNA, contain numerous functional signals of various origin and purpose. They include thousands of non-protein coding RNAs [2], numerous gene expression regulatory signals that surround each gene, chromatin folding structures which include nucleosome positioning sites and scaffold/matrix attached regions $[3,4]$. These functional DNA regions are non-random in their genomic sequence. The non-randomness or inhomogeneity of base composition has been described at different levels of complexity and sequence length. Starting on the short scale, inhomogeneity occurs in the non-random associations of neighboring bases with each other [5], through the over and under-abundance of particular "words" (usually 5-10 base long oligonucleotides) [6] or longer stretches of DNA, also known as "pyknons" ( 18 bases long) [7,8], and up to large regions that cover hundreds of thousands of nucleotides [9]. Compositional inhomogeneity is known to exist in all kinds of species from bacteria to human. However, the particular arrangement of such sequence patterns is often species-specific [10].

It has been the focus of our research to elucidate the genomic sequence non-randomness that we call MidRange Inhomogeneity or MRI [11]. We define MRI to be genomic regions from $30 \mathrm{bp}$ to several thousand nucleotides with particular nucleotide enrichments. For large mammalian genomes, there is a high probability that a random sequence of length 20 nucleotides will be unique. Thus, for examining mid-range genomic signals we do not look at particular "words" but only the overall compositional content of particular base(s) that we refer to as $X(X$ could be a single nucleotide $\mathrm{A}, \mathrm{G}, \mathrm{C}$, or $\mathrm{T}$ or any of their combinations like $\mathrm{A}+\mathrm{C}$, or $\mathrm{G}+\mathrm{T}+\mathrm{C}$ ). We created a public Internet resource, "Genomic MRI" to study the distribution of $X$-rich regions in any sequence of interest. It was demonstrated that $X$-rich MRI regions are highly overrepresented in mammalian genomes for all kinds $X$-contexts. Particular properties of MRI have also been investigated previously by Mrazek and Kypr [12] and also by Nikolaou and Almirantis [13]. This paper studies the effect of mutations on the evolution of MRI regions in primates.

\section{Results}

\section{Substitution and polymorphism inside $M R I$ regions}

¿From the whole-genome human-chimp-macaque alignment we extracted all the aligned sequences with inhomogeneous nucleotide compositions that satisfy the criteria for MRI (so-called X-rich MRI regions; see the Materials and Methods section) and also control regions with nucleotide compositions equal to the average values for the entire human genome. We used the default MRI region length of 100 nucleotides for all computations. Only SNPs located within these MRI and control regions were studied. We particularly focused on the single nucleotide substitutions that maintain or erode MRI features. For example, in GT-rich MRI regions we counted the total number of novel polymorphisms that erode the feature, i.e. $\mathrm{G}$ or $\mathrm{T} \rightarrow \mathrm{C}$ or A substitutions, denoted as $N_{G T \rightarrow C A}$ and also the total number of those that maintain the MRI features, i.e. $\mathrm{C}$ or $\mathrm{A} \rightarrow \mathrm{G}$ or $\mathrm{T}$ substitutions, denoted as $N_{C A \rightarrow G T}$. In addition, the entire set of recent human substitutions; that is, those nucleotides that differed in human but were the same in chimp and macaque, were processed for the MRI and control regions and presented as "fixed substitutions". The substitution ratio, $S_{X}$ (recall that: $S_{X}=$ $N_{X \rightarrow \text { nonX }} / N_{\text {nonX } \rightarrow X}$ ) for the numbers of substitutions that maintain and erode $X$-rich MRI features was calculated for each substitution subtype (rare, minor, medium, and major SNPs and 'fixed'--refer to the Methods section for a detailed explanation) and presented in Figures 1 and 2. With respect to $X$-rich or poor MRI regions, the $X$ in Figure 1 represents a two base combination such as GC, AG, GT, etc. while in Figure $2 X$ can be any single nucleotide, e.g., A, T, C, and G. If the $S_{X}$-ratio is equal to 1 the $X$-rich region does not tend towards a change in its $X$-base composition. When $S_{X}>1$, the substitutions reduce the $X$-richness of the examined regions, whereas when $S_{X}<1$, substitution rates elevate the $X$-richness of the regions. Figures 1 and 2 demonstrate clear linear trends for $S_{X}$-ratios with respect to increasing fixation of substitutions within human populations.

For the cases of GT-, AC-, AG-, and TC-rich MRI regions (Figure 1), all $S$-ratios for rare SNPs are close to 1.8 (showing erosion of the MRI features). For major SNPs and fixed mutations the $S_{G T}$ and $S_{A C}$-ratios reach 1.0 (which means no change in the corresponding base composition) and $S_{G A}$ and $S_{C T}$-ratios reach 1.2 respectively. As for the corresponding control GT-, AC-, AG-, and TC-average regions (all having $50 \%$ of corresponding base composition) these lines are flat with all $S$-ratios equal to 1 . The latter result is highly expected because of the symmetry of $(+)$ and (-) chromosomal strands for these particular base compositions. Figure 1 also demonstrates that in GC-rich MRI regions the $S_{G C}$-ratio change has the highest slope from 7.0 for rare SNPs to 1.6 for fixed substitutions. In ATrich MRI regions (also referred to as nonGC-rich in the tables) the change of $S_{A T}$-ratio has the lowest slope starting from 1.7 (rare SNPs) and ending at 1.3 (fixed substitutions). The control regions with the average GC/AT compositions (40-42\% GC and 58-60\% AT) also demonstrate a clear change of S-ratios during substitution fixation. In the control GC-average regions, rare SNPs favor 

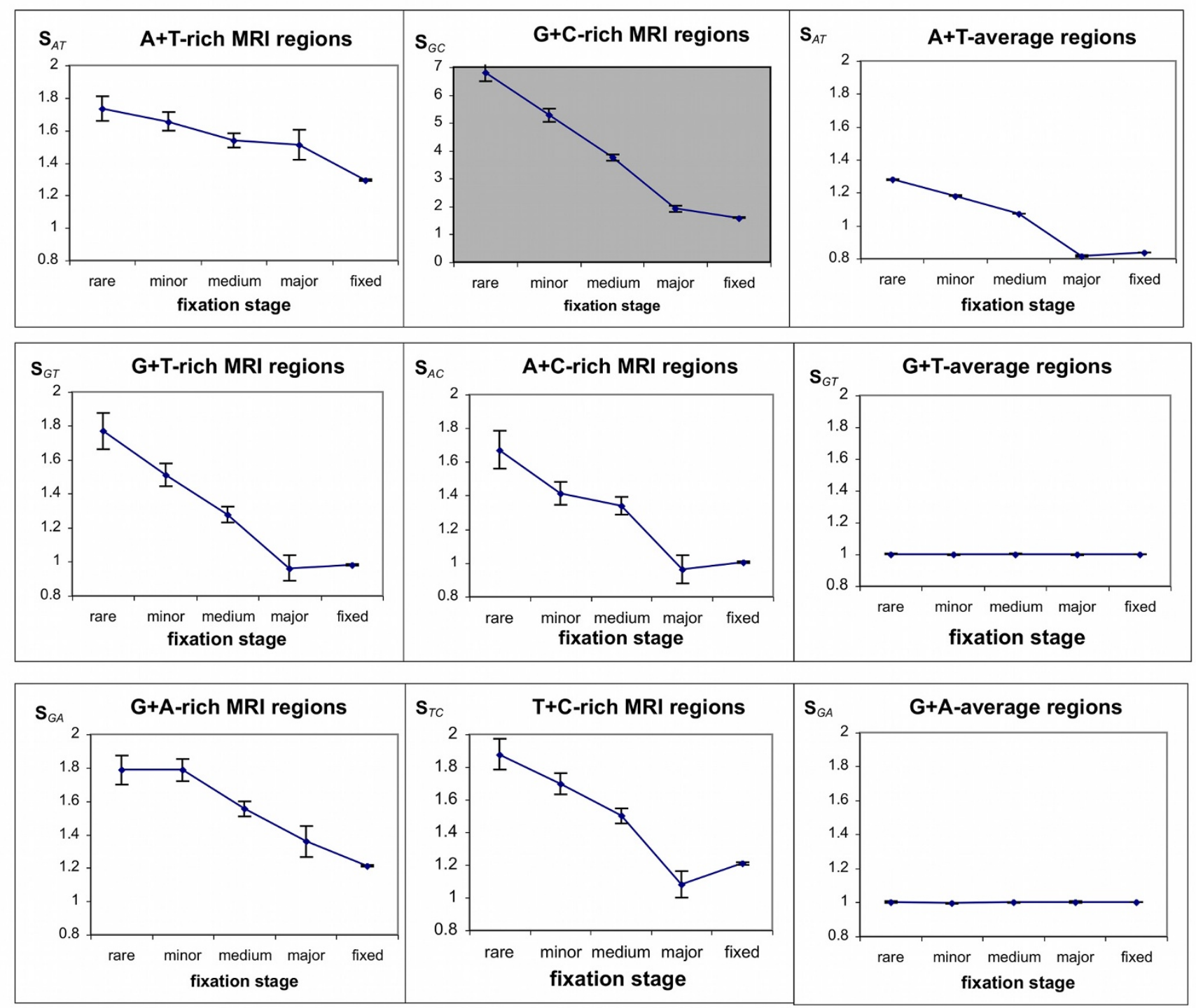

Figure I

Substitution Rates in MRI Regions for a Combination of Nucleotides. For each X MRI region--where $X$ is for GC-, GT-, or GA-rich or poor regions--the $X$-base composition rate of change is given for all substitutions at different levels of fixation within the human population. The rate of change $\left(S_{X}\right)$ is the ratio of $X$ to non $X$ substitutions over non $X$ to $X$ substitutions in those particular $X$-rich regions. Thus, a ratio of I means no change in the $X$-richness of the region whereas a ratio greater than I implies degradation of the $X$-rich region and less than I implies enrichment of the $X$-rich MRI region. Note that in the control $X$-average regions the $S_{X}$-ratio is always inverse to $S_{\text {non } X}$-ratio $\left(S_{X}=I / S_{\text {nonX }}\right)$. Therefore, only one graph for each $S_{X}$ and $S_{\text {nonX } X}$ pair is presented. Since there are significant variations in $S_{X}$-ratios for different $X$ compositions, the graphs are presented in two different scales. The white background presents changes of $S_{X}$-ratios in the 0.8 to 2 range, while the gray background presents changes in the 0 to 7 range. Vertical bars show the standard error of the means (see Methods section).

increasing AT-richness $\left(S_{G C}\right.$-ratio of 1.3$)$ whereas fixed mutations demonstrate the opposite effect $\left(S_{G C}\right.$-ratio of $0.8)$.

The data for the $S$-ratios for single nucleotides (Figure 2) are very similar to the trends seen in GC- and AT-rich regions. As expected from (+/-) strand symmetry, $S_{G}$-ratios are equal to $S_{C}$-ratios and represent about a half of the GC trend. The minor differences between G- and C-rich regions are within the errors of measurement. In the same way the $S_{A}$-ratios are seen to be the same as the $S_{T}$-ratios and they comprise approximately half of the effect seen for AT-rich regions. 

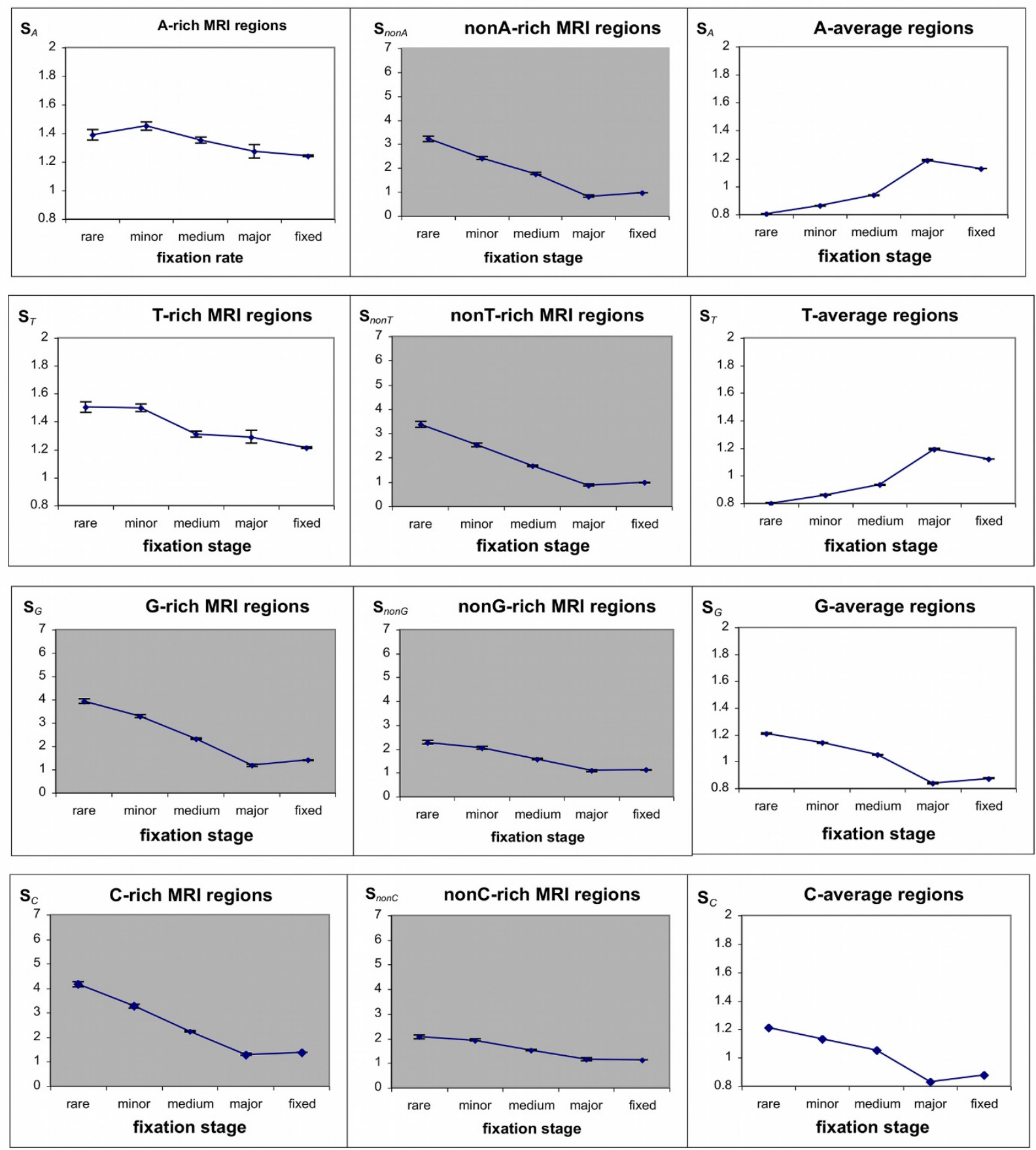

Figure 2

Substitution Rates in MRI Regions for Single Nucleotides. For each $X$ MRI region--where $X$ is for A-, T-, G-, or C-rich or poor regions--the $X$-base composition rate of change is given for all substitutions at different levels of fixation within the human population. The rate of change $\left(S_{X}\right)$ is the ratio of $X$ to non $X$ substitutions over non $X$ to $X$ substitutions in those particular $X$-rich regions. Thus, a ratio of I means no change in the $X$-richness of the region whereas a ratio greater than I implies degradation of the $X$-rich region and less than $I$ implies enrichment of the $X$-rich MRI region. Note that in the control $X$-average regions the $S_{X}$-ratio is always inverse to $S_{\text {non } X \text {-ratio }}\left(S X=I / S_{\text {nonX }}\right)$. Therefore, only one graph for each $S X$ and $S_{\text {non } X \text { pair is }}$ presented. Since there are significant variations in $S_{X}$-ratios for different $X$ compositions, the graphs are presented in two different scales. The white background presents changes of $S_{X}$-ratios in the 0.8 to 2 range, while the gray background presents changes in the 0 to 7 range. Vertical bars show the standard error of the means (see Methods section) 
Based on the observed $S_{X}$-ratios and the current percentage of $X$ bases in the genomic regions under investigation, we calculated the projected equilibrium composition representing the future $X$-composition toward which the examined substitution rates drive these regions. In other words, the equilibrium $X$-composition shows the future level of $X$-richness that would be approached if the $S_{X^{-}}$ ratio as it is observed now were maintained indefinitely. The computed equilibria for each subgroup of substitutions are presented in Table 1. For instance, in the GA-rich regions $(\mathrm{G}+\mathrm{A}$ composition of $70 \%$ ), rare SNPs drive GArichness of these MRI regions down to an equilibrium of $56.6 \%$, while nearly fixed or fixed substitutions drive the GA-composition only to the $65.8 \%$ level. For each type of $X$-rich MRI, there is a trend toward minimizing the damage of mutations and preserving the MRI feature as the fixation of the observed substitutions increases. The highest preservation effect is seen for GT- and AC-rich regions (with an observed $X$-base composition of 70\%), where the equilibria for fixed substitutions reach about the same level of $70 \%$. For the rest of the types of MRI regions, their equilibria composition is a little below the currently observed base composition.

In order to estimate mutation rates for MRI regions versus their respective control regions, we counted the occurrence rates for rare SNPs. The frequency ratio of rare SNPs in MRI rich regions to those in the control regions was calculated. The smallest ratio observed was for $\mathrm{A}+\mathrm{C}$ content $(0.464)$. This means the frequency of rare SNPs within MRI AC-rich regions is approximately half that of control regions. The highest occurrence ratio for rare SNPs was observed in G- and C-rich MRI regions (1.16 and 1.17 respecitvely). Thus, the occurrence rates of rare SNPs is slightly lower in MRI regions than in the corresponding control regions with the exception of G- and C-rich MRI regions. The entire dataset for the SNPs occurrences in MRI and control regions is presented in Additional file 1. The prevalence of rare and minor SNPs over major SNPs was also observed, their proportion over every MRI and control regions being 5.79 .

Table I: Projected X-Equilibria.

\begin{tabular}{|c|c|c|c|c|c|c|}
\hline \multirow[b]{2}{*}{ Type of region } & \multirow[b]{2}{*}{ Observed X-percentage } & \multirow[b]{2}{*}{ rare SNPs } & \multicolumn{4}{|c|}{ Equilibrium for $X$-percentage computed from each substitution rate } \\
\hline & & & minor SNPs & Medium SNPs & major SNPs & fixed substit. \\
\hline G-rich & $40 \%$ & $14.5 \%$ & $16.9 \%$ & $22.3 \%$ & $36.0 \%$ & $32.1 \%$ \\
\hline nonG-rich & $7 \%$ & 14.6 & 13.4 & 10.6 & 7.6 & 7.8 \\
\hline G-average & $20 \%$ & 17.1 & 18.0 & 19.2 & 23.0 & 22.2 \\
\hline C-rich & $40 \%$ & 13.8 & 16.9 & 23.0 & 33.90 & 32.4 \\
\hline nonC-rich & $7 \%$ & 14.5 & 12.7 & 10.2 & 8.0 & 7.8 \\
\hline C-average & $20 \%$ & 17.1 & 18.1 & 19.2 & 23.1 & 22.1 \\
\hline A-rich & $49.5 \%$ & 41.4 & 40.3 & 42.0 & 43.5 & 44.1 \\
\hline nonA-rich & $12.9 \%$ & 32.3 & 26.3 & 20.8 & 10.9 & 12.6 \\
\hline A-average & $29.4 \%$ & 34.1 & 32.6 & 30.7 & 26.0 & 27.0 \\
\hline T-rich & $49.5 \%$ & 39.5 & 39.5 & 42.8 & 43.2 & 44.6 \\
\hline nonT-rich & $12.9 \%$ & 33.4 & 27.3 & 19.9 & 11.5 & 12.6 \\
\hline T-average & $29.4 \%$ & 34.2 & 32.6 & 30.8 & 25.9 & 27.1 \\
\hline GT-rich & $69.8 \%$ & 56.9 & 60.7 & 64.6 & 70.8 & 70.4 \\
\hline nonGT-rich & $30.1 \%$ & 41.7 & 37.7 & 36.5 & 29.2 & 30.1 \\
\hline GT-average & $50.0 \%$ & 49.9 & 50.0 & 50.0 & 50.0 & 50.0 \\
\hline GA-rich & $70.0 \%$ & 56.6 & 56.6 & 60.0 & 63.2 & 65.8 \\
\hline nonGA-rich & $29.9 \%$ & 44.6 & 42.1 & 39.1 & 31.7 & 34.1 \\
\hline GA-average & $50.0 \%$ & 49.9 & 50.1 & 50.0 & 49.9 & 49.9 \\
\hline GC-rich & $71.3 \%$ & 26.4 & 31.7 & 39.5 & 56.1 & 60.6 \\
\hline nonGC-rich & $20.0 \%$ & 30.2 & 29.3 & 27.8 & 27.4 & 24.4 \\
\hline GC-average & $40.7 \%$ & 34.9 & 36.8 & 39.0 & 45.7 & 45.0 \\
\hline
\end{tabular}

The calculated equilibria percentages (see Equation 3) for $X$-bases in $X$-rich MRI and control regions with average $X$-composition. Projected equilibria are given based on the substitution rates of rare, minor, medium, and major SNPs as well as for the fixed substitution rates (chimpmacaque to human). 


\section{Insertions and deletions inside $M R I$ regions}

Using the same computational approach as for substitutions, we analyzed human-chimp-macaque triple alignments for the characterization of indels (insertions \& deletions) that occurred in the human genome during the last 10 million years after the divergence of $H$. sapiens and $P$. troglodytes species. We particularly investigated how indels change the nucleotide composition of MRI regions and control regions with average nucleotide composition. The complete set of data representing short indels (whose sizes are less than three nucleotides) and medium indels (whose sizes are from three to fifty nucleotides) is presented in Additional file 2 (S2A--contains MRI for combinations of nts; S2B--MRI for individual nts). Large indels with sizes over 50 bp were not examined since they are comparable with the sizes of MRI regions and, thus, compromise proper characterization of MRI. The summary data on the influence of both short and medium indels on the composition of MRI and control regions are presented in the Tables 2 and 3 (Table 2 shows MRI regions where $X$ represents any single nucleotide; Table 3 is for when $X$ represents any combination of 2 nucleotides). For each type of $X$-rich and $X$-control regions the total number of inserted and deleted $X$ and nonX nucleotides have been computed: $N_{\text {ins }(X)}, N_{\text {ins }(n o n X)}, N_{\text {del }(X)}, N_{\text {del(nonX) }}$. Finally, the net change in $X$ and non $X$ compositions due to indels have been calculated using the following formulas:

$$
\begin{gathered}
\Delta X=N_{i n s(X)}-N_{\operatorname{del}(X)} \\
\Delta n o n X=N_{i n s(\text { non } X)}-N_{\operatorname{del}(\operatorname{non} X)}
\end{gathered}
$$

Tables 2 and 3 demonstrate that in the human genome there is a prevalence of deletions over insertions (i.e. negative values of $\Delta X$ and $\Delta n o n X$ ) for every type of nucleotide content studied and for every type of MRI and control region with the exception of GC-indels in GC-rich MRI regions. In the last case $\Delta \mathrm{GC}$ is positive and equal to 1405 added nucleotides (over a total set of 1.8 million nucleotides). For all other cases of $X$ except $X=\mathrm{GC}$, short and medium indels cause gradual contraction of genomic regions in humans. This means that there is no nucleotide composition equilibrium to which the indels drive the genome in the indefinite future and, therefore, these equilibria have not been calculated. Table 2 shows that, for every $X$-rich region, indels result in the increasing the richness of corresponding MRI regions (positive net $X \%$ change for $X$-rich region and negative net $X \%$ change for non $X$-rich region). In all $X$-control regions the net $X \%$ change is several times less than in the corresponding $X$ rich and nonX-rich regions.

Finally, we calculated the percentage of nucleotide composition changes in case of both substitutions and indels separately, that occurred in the human genome during
Table 2: Impact of Indels on X-rich MRI Regions, with $X$ Representing Any Single Base. The impact of indels on $X$-rich MRI regions and on $X$-average regions, where $X$ is for $A-$, T-, $C$-, or G-rich or poor. For each particular region we give the total length of examined regions in mega-bases, the percentage composition or content of $X$, the number of changes in $X$ due to insertions and deletions $\left(\Delta X=N_{\text {ins }}(X)-N_{\text {del }}(X)\right)$, and the change in

\begin{tabular}{|c|c|c|c|}
\hline & A-rich & nonA-rich & A-average \\
\hline total length & $66.9 \mathrm{Mb}$ & $72.4 \mathrm{Mb}$ & $800.4 \mathrm{Mb}$ \\
\hline content of $A$ & $49.6 \%$ & $12.9 \%$ & $30.5 \%$ \\
\hline$\Delta \mathrm{A}$ & -16850 & -7390 & -44182 \\
\hline$\Delta$ nonA & -24748 & -29257 & -98769 \\
\hline net $\mathrm{A} \%$ change INDEL & $0.006 \%$ & $-0.004 \%$ & $-0.0001 \%$ \\
\hline \multirow[t]{2}{*}{ net $A \%$ change SUBST } & $-0.027 \%$ & $-0.002 \%$ & $-0.014 \%$ \\
\hline & T-rich & nonT-rich & T-average \\
\hline total length & $67.8 \mathrm{Mb}$ & $71.1 \mathrm{Mb}$ & $800.4 \mathrm{Mb}$ \\
\hline content of $\mathrm{T}$ & $49.5 \%$ & $13.1 \%$ & $30.5 \%$ \\
\hline$\Delta \mathrm{T}$ & -21849 & -7078 & -47238 \\
\hline$\Delta$ nonT & -24084 & -22716 & -97057 \\
\hline net T\% change INDEL & $0.001 \%$ & $-0.004 \%$ & $-0.0004 \%$ \\
\hline \multirow[t]{2}{*}{ net T\% change SUBST } & $-0.024 \%$ & $-0.002 \%$ & $-0.013 \%$ \\
\hline & G-rich & nonG-rich & G-average \\
\hline total length & $52.0 \mathrm{Mb}$ & $60.4 \mathrm{Mb}$ & $884.7 \mathrm{Mb}$ \\
\hline content of G & $40.10 \%$ & $7.20 \%$ & $20.40 \%$ \\
\hline$\Delta \mathrm{G}$ & -1185 & -7080 & -31780 \\
\hline$\Delta$ nonG & -12864 & -37512 & -139126 \\
\hline net G\% change INDEL & $0.009 \%$ & $-0.006 \%$ & $0.0003 \%$ \\
\hline \multirow[t]{2}{*}{ net G\% change SUBST } & $-0.052 \%$ & $0.009 \%$ & $0.016 \%$ \\
\hline & C-rich & nonC-rich & C-average \\
\hline total length & $52.0 \mathrm{Mb}$ & $60.4 \mathrm{Mb}$ & $883.9 \mathrm{Mb}$ \\
\hline content of $C$ & $40.10 \%$ & $7.20 \%$ & $20.50 \%$ \\
\hline$\Delta \mathrm{C}$ & -829 & -6700 & -33823 \\
\hline$\Delta$ nonC & -12418 & -35277 & $-|4033|$ \\
\hline net C\% change INDEL & $0.009 \%$ & $-0.006 \%$ & $0.0002 \%$ \\
\hline net C\% change SUBST & $-0.049 \%$ & $0.009 \%$ & $0.015 \%$ \\
\hline
\end{tabular}
$X$ composition due to both indels and substitutions.

last ten million years after the divergence of human and chimpanzee. These results are presented in Tables 2 and 3 and serve to measure the relative importance of substitutions versus indels to the nucleotide composition of MRI regions.

\section{Discussion}

Consistent with Chargaff's second parity rule [14], both the $\mathrm{G}$ or $\mathrm{C}$ base content of the human genome are equal to $21.1 \%$, while A or T comprise $28.9 \%$ each. However, in thousands and thousands of genomic regions of various lengths, the composition of A, T, C, or G content (or different combinations of these bases) exist at extremes quite different from the aforementioned averages. De novo 
Table 3: Impact of Indels on MRI Regions, with $X$ Representing Combinations of Any Two Bases.

\begin{tabular}{|c|c|c|c|}
\hline & GC-rich & nonGC-rich & GC-average \\
\hline total length & $17.8 \mathrm{Mb}$ & $54.8 \mathrm{Mb}$ & $780.6 \mathrm{Mb}$ \\
\hline content of GC & $71.00 \%$ & $20.30 \%$ & $40.90 \%$ \\
\hline$\Delta \mathrm{GC}$ & 1405 & -9100 & -31622 \\
\hline$\Delta$ nonGC & -765 & -5951 & -56278 \\
\hline net GC\% change INDEL & $0.005 \%$ & $-0.011 \%$ & $0.001 \%$ \\
\hline \multirow[t]{2}{*}{ net GC\% change SUBST } & $-0.094 \%$ & $0.042 \%$ & $0.034 \%$ \\
\hline & GT-rich & nonGT-rich & GT-average \\
\hline total length & $34.9 \mathrm{Mb}$ & $34.6 \mathrm{Mb}$ & $1192 \mathrm{Mb}$ \\
\hline content of GT & $69.10 \%$ & $30.90 \%$ & $50.00 \%$ \\
\hline$\Delta \mathrm{GT}$ & -8278 & -6837 & -121644 \\
\hline$\Delta$ nonGT & -4518 & -8502 & -120128 \\
\hline net GT\% change INDEL & $0.002 \%$ & $-0.006 \%$ & $-0.0001 \%$ \\
\hline \multirow[t]{2}{*}{ net GT\% change SUBST } & $0.004 \%$ & $0.001 \%$ & $-0.0003 \%$ \\
\hline & GA-rich & nonGA-rich & GA-average \\
\hline total length & $69.2 \mathrm{Mb}$ & $70.0 \mathrm{Mb}$ & $978.3 \mathrm{Mb}$ \\
\hline content of GA & $69.75 \%$ & $30.22 \%$ & $49.99 \%$ \\
\hline$\Delta \mathrm{GA}$ & $-2364 I$ & -13935 & -96617 \\
\hline$\Delta$ nonGA & -14185 & -28480 & -100013 \\
\hline net GA\% change INDEL & $0.004 \%$ & $-0.002 \%$ & $0.0002 \%$ \\
\hline net GA\% change SUBST & $-0.014 \%$ & $0.014 \%$ & $0.0002 \%$ \\
\hline
\end{tabular}

The impact of indels on $X$-rich MRI regions and on $X$-average regions, where $X$ is for GC-, GT-, or GA-rich or poor. For each particular region we give the total length of examined regions in mega-bases, the percentage composition or content of $X$, the number of changes in $X$ due to insertions and deletions $\left(\Delta X=N_{\text {ins }}(X)-N_{\text {del }}(X)\right)$, and the net change in $X$ composition due to both indels and substitutions.

mutations constantly occur in populations and could dramatically change the base composition of a genomic region during the course of evolution. A good choice for a large-scale computational analysis of these novel mutations is in the examination of 'rare' single-nucleotide polymorphisms (SNPs, or mutations that are present only in a small group of individuals and absent in a majority of the population). Rare SNPs are mutations that have recently occurred. However, even among rare SNPs there exists a minor subgroup of "older" mutations that have diminished their frequency to rare events. The relative size of this subgroup is in reverse proportion to the effective size of the population [15], and hence, it represents only a minor fraction of the recent mutations for humans. Here we show that rare SNPs in genomic regions with average nucleotide composition are enriched by $\mathrm{G}$ or $\mathrm{C} \rightarrow \mathrm{T}$ or $\mathrm{A}$ substitutions that drive the genomic composition of those regions to a level of $35 \%$ for $\mathrm{G}+\mathrm{C}$ and $65 \%$ for $\mathrm{A}+\mathrm{T}$. On the other hand, examining the same regions for mutations that have substantially propagated into human populations (i.e. medium and high frequency SNPs as well as "fixed" recent mutations) demonstrates that these fixed or nearly fixed substitutions are much less prone to $\mathrm{G}$ or $\mathrm{C} \rightarrow$ $\mathrm{T}$ or A changes. Instead, high frequency SNPs as well as fixed substitutions tend to drive genomic regions with average base composition to $45 \% \mathrm{G}+\mathrm{C}$ composition.

Here we have focused particularly on the influence of mutations on the evolution of specific genomic regions with strongly inhomogeneous base compositions that are far from the average distribution of nucleotides (so-called MRI regions where $\mathrm{G}+\mathrm{C}, \mathrm{G}+\mathrm{A}, \mathrm{C}+\mathrm{T}, \mathrm{G}+\mathrm{T}$, or $\mathrm{A}+\mathrm{C}$ composition is at least $70 \%, \mathrm{~A}+\mathrm{T}$ composition is above $80 \%$, or single base frequency reaches nearly $50 \%$ ). For all types of MRI regions, we found that novel substitutions (rare SNPs) tend to more strongly erode the compositional extremes ( $X$-richness) of the region. At the same time, these mutations undergo a strong fixation bias during their propagation into populations in such a way that fixed substitutions tend to preserve MRI regions. For example, rare SNPs inside GC-rich MRI regions drive the nucleotide composition of those regions to the $26 \%$ GC level. However, fixed substitutions in the same GC-rich MRI regions drive GC composition only to $61 \%$. The highest fixation was seen for GT- and AC-rich MRI regions, which preserves the current GT- and AC-composition of $70 \%$.

This trend of preserving nucleotide composition of MRI regions with respect to the increasing fixation of substitutions could be explained by at least two different mechanisms. First, one could observe that there are some important functional roles for MRI regions. For instance, GC-rich MRI regions include well-known CG-islands, prominent regulators for gene expression [16,17]. Thus, these regions should be under the constraint of purifying selection, preserving their important features. Other MRI regions may be under similar selective pressure due to association with functional genomic elements and/or, as yet unknown, sequence signals. Second, fixation bias inside MRI regions might be due to some non-symmetry in cellular molecular machinery involving DNA repair, replication, and/or recombination processes. For example, the Biased Gene Conversion (BGC)-theory engages this particular scenario in order to explain the maintenance of CG-rich regions $[18,19]$. (It must be observed, however, that this theory operates on much larger genomic scales and refers to isochores that cover from hundreds of thousands to millions of bases.) Thus far it is inconclusive as to which of these two scenarios, or a combination thereof, best fits the observed trends. For the case of GC-rich sequences, we conjecture that both scenarios could be taking place to some extent to preserve MRI.

Interestingly, the highest level of MRI erosion for rare SNPs is observed in GC-rich MRI regions. Novel substitutions in these particular regions try to drive GC-content to 
the lowest level of $26 \%$ (see Table 1). We explain this phenomenon via uneven distribution of $\mathrm{CpG}$ dinucleotides, which are most abundant in GC-rich MRI regions. It is well known that $\mathrm{CpG}$ dinucleotides are extreme hot spots for the $\mathrm{C} \rightarrow \mathrm{T}$ and $\mathrm{G} \rightarrow$ A mutations, which cause $\mathrm{CpG}$ to be the most underrepresented dinucleotide in vertebrate genomes. Therefore, CG-rich MRI regions, which are known to have the highest concentration of CpG dinucleotides, should have the highest rate of de novo mutations in the direction $\mathrm{C}$ or $\mathrm{G} \rightarrow \mathrm{T}$ or A. Human SNPs having $\mathrm{C} /$ $\mathrm{T}$ alleles in the $\mathrm{CpG} / \mathrm{TpG}$ context with the orthologous chimp allele in the TpG context have an increased error rate of $9.8 \%$ for ancestral misidentification (see the Methods section) due to the probability of a coinciding chimp SNP at the same locus [20]. However, since the strength of the mutational erosion in the GC-rich MRI regions is so high, even an error rate of $9.8 \%$ will not change the observed trend.

So far we have discussed only the effect of substitutions on the nucleotide composition of mid-range genomic regions. Insertions and deletions are the other types of mutations that change genomic sequences and, therefore, should also be considered. In mammals, short and medium indels are several times less frequent than substitutions. Currently, there is not enough data on human indel SNPs to perform the same analysis of their fixation process as we did for substitutions. For this reason we studied only fixed indels in humans (indels present in human but differing in chimp and macaque). Our examination demonstrated that indels weakly influence the nucleotide content of MRI regions toward preserving their inhomogeneous composition, in the same manner as the fixation bias of fixed substitutions (see Tables 2 and 3 ).

\section{Conclusion}

The fixation bias on both fixed substitutions and indels tend to protect MRI regions from degradation of their compositional extremes amid the constant flow of random mutations, thus suggesting their contribution in the preservation of functional and structural complexities of the human genome. Future research on these genomic elements as well as refinement of our approach should help determine the extent of maintenance of MRI by natural selection.

\section{Methods}

\section{Genomic samples and computation of recent human mutations ("fixed substitutions")}

Taking human-chimp (human build 36.1 and chimp build 2 version 1 ) and human-macaque (macaque build v1 edit4) whole-genome pairwise alignments from the UCSC Genome Browser [21] http://hgdown load.cse.ucsc.edu/downloads.html as input, we generated a Perl script for the identification of the common genomic regions for these three species. The process involved the usage of the human genomic sequence as the reference for the location with the chimp and macaque sequences being extracted only in areas where the sequences of all three species were represented. We then invoked the ClustalW (v1.83) program with default parameters to obtain a whole-genome human-chimpanzee-macaque triple alignment. The obtained alignment is available at our website http://bpg.utoledo.edu/human_chimp_macaque.html.

This triple alignment was used to calculate the dataset of recent mutations in humans. We considered a recent substitution at a particular position (for example $\mathrm{T} \rightarrow \mathrm{C}$ at position 23456719 on chromosome 7) to be valid if the human genome has a $\mathrm{C}$ base while both chimp and macaque have a $\mathrm{T}$ base in the corresponding aligned positions. In addition, we required that the quality of the alignment in the vicinity of the mutation be reliable (more than 70\% similarity between human and macaque in the $20 \mathrm{bp}$ flanking region $[-10,+10])$. The frequency table of all inferred recent human mutations is presented in the Additional file 3 . We analyzed these recent substitutions together with the SNP datasets and call the former mutations "fixed substitutions," assuming that the majority of them occurred less than 10 million years ago and were already fixed across all human populations. In the same manner we processed indels in the triple alignments and computed all unambiguous cases of human insertions and deletions with sizes from 1 to 49 nucleotides.

\section{Processing of SNP data}

Over 4.62 million human SNPs from all chromosomes were obtained (dbSNP build 128 [22], ftp:// ftp.ncbi.nih.gov/snp/), filtered for completeness and correctness annotations (676499 records discarded total), and mapped onto the whole-genome human-chimpanzee alignment. SNP allele frequencies were averaged from the frequency data of all populations of that allele. However, only those SNPs that were successfully located within the alignment were processed further. For each SNP site we verified the existence of the particular polymorphic bases in the specified position of the human genome reference sequence and also in the corresponding aligned position on the chimp genomic sequence. If any of these two species had different bases than the SNP alleles, the SNP was discarded (20469 SNPs discarded total).

Otherwise, we defined the origin of the polymorphism based on the chimpanzee nucleotide. Consider the following example to illustrate this process: suppose one has an A/G polymorphism located at position 34567812 of chromosome 5 with an average A allele frequency of 0.6 and a $G$ allele frequency of 0.4. Then at position 34567812 of chromosome 5 of the human genome reference sequence (Genbank build 36.1), we would first examine if the $\mathrm{A}$ or $\mathrm{G}$ allele is present at that position and 
discard the SNP if not. Next, using the flanking region of that SNP we could align the chimp genomic sequence. If the chimp nucleotide were T or $\mathrm{C}$ then the SNP would also be discarded because those alleles are not a part of the human haplotype at that position. However supposing that the chimp nucleotide were $\mathrm{G}$, then the polymorphism would be declared as a $\mathrm{G} \rightarrow$ A polymorphism with $\mathrm{G}$ being declared the ancestral allele that at some point in human evolution mutated into an A allele within some human population(s). From the frequency data we may finally characterize this example SNP more precisely as a $0.4 \mathrm{G} \rightarrow 0.6 \mathrm{~A}$ polymorphism.

Using this approach we successfully characterized 3.93 million human SNPs. This last group of SNPs was divided into four subgroups based on the abundance of the mutant allele in the given human populations:

I. rare polymorphisms with the frequency of the mutated allele being less than 3\%;

II. minor polymorphisms with frequencies ranging from $3 \%$ to $20 \%$;

III. medium polymorphisms with frequencies going from $20 \%$ to $80 \%$; and

IV. major polymorphisms with the frequency being above $80 \%$.

For our method, misidentification of the ancestral allele might arise when the site for the human SNP is also polymorphic in chimp populations (e.g. A/G polymorphism) or for the possible case that this site had a recent substitution in chimps $(A \rightarrow G)$ after their divergence from humans. Human and chimpanzee genomes only differ by $1.23 \%$ due to single nucleotide substitutions with $1.06 \%$ being due to fixed substitutions and the rest $(0.17 \%)$ being due to polymorphisms in human and chimp [20]. Moreover, according to the Chimpanzee Sequencing and Analysis Consortium the average estimated error rate of human alleles being misidentified due to chimp polymorphisms is only $\sim 1.6 \%$ across all typical SNPs, which is acceptably low. It is also observed, however, that in the mutational hotspot of the CpG dinucleotide, there is an increased error rate for ancestral misidentification. If the human alleles are $\mathrm{C} / \mathrm{T}$ in the $\mathrm{CpG}$ and $\mathrm{TpG}$ context and the chimp allele is $\mathrm{T}$ (in the TpG context) then the estimated error rate is actually $9.8 \%$ [20]. Thus, in the context of studying our MRI regions, any substitution (especially in GC-rich MRI regions since they contain an overabundance of $\mathrm{G}$ and $\mathrm{C}$ ) going from TpG $\rightarrow \mathrm{CpG}$ could have the ancestral allele misidentified, which would mean that the substitution would actually be $\mathrm{CpG} \rightarrow \mathrm{TpG}$, although in the case of GC-rich MRI regions where such dinucleotides are more likely, an error rate of $9.8 \%$ is not sufficient to change the trend or conclusion of our results.

\section{X-rich MRI genomic regions and control regions with average base composition}

Any base or combination of bases can be described by a parameter $X$. For example, $X$ could be G-base; C+T-bases; or $\mathrm{A}+\mathrm{T}+\mathrm{G}$ bases, et cetera. It is also useful to refer to nonX base(s) as all bases not $X$. Thus, $X+$ non $X$ must represent all four nucleotides $A, G, T$, and $C$. For the examples above, nonX are $\mathrm{A}+\mathrm{T}+\mathrm{C}$-bases; $\mathrm{G}+\mathrm{A}$-bases, and $\mathrm{C}$-base, respectively. MRI is characterized by a specific base composition within a region under analysis. We characterize $X$-rich MRI regions based on an overabundance of the $X$ base(s) within a region of a certain length (the so-called window), where the percentage of $X$ should be above a certain threshold (Bechtel et al 2008). We calculated MRI regions in the human genome for single nucleotides and various nucleotide combinations using a stretchy window of 100+ nucleotides with the following threshold parameters: for A or T the threshold was $49 \%$; for $\mathrm{G}$ or $\mathrm{C}$ we used $40 \%$; for $\mathrm{G}+\mathrm{C}$ it was $70 \%$; for $\mathrm{A}+\mathrm{T}$ the threshold was at $80 \%$; for $\mathrm{G}+\mathrm{T}, \mathrm{C}+\mathrm{A}, \mathrm{G}+\mathrm{A}$, and $\mathrm{C}+\mathrm{T}$ were at $70 \%$; nonA or nonT was $87 \%$; and non $\mathrm{G}$ or non $\mathrm{C}$ the threshold was 93\%. These thresholds were chosen experimentally in such a way that MRI regions should represent about $2 \%$ of the whole human genome. A stretchy window of $N+$ nucleotides means that we scan genomic sequence with an $\mathrm{N}$-size window to find a genomic MRI region that fits the threshold criterion, then we extend the window above the detected region by $10 \mathrm{nt}$ steps until the criterion is no longer met. After registering the full MRI region we jump beyond the current MRI region and continue with the default $\mathrm{N}$-size window. Using this approach we characterized all MRI regions in the triple human-chimp-macaque alignments using the human sequence for calculating nucleotide composition and MRI features. We also discarded those MRI regions in the alignments where the indel composition exceeded 50\%. For the collection of control regions with average base compositions we used the same stretchy window approach with the nucleotide composition corresponding to the following average genomic frequencies: for $\mathrm{A}$, T between 30 and $31 \%$ thresholds; for $\mathrm{G}$, C between $20-21 \%$; for $\mathrm{G}+\mathrm{C}$ between $40-42 \%$; $\mathrm{A}+\mathrm{T}$ at $58-60 \% ; \mathrm{G}+\mathrm{T}, \mathrm{C}+\mathrm{A}, \mathrm{G}+\mathrm{A}$, or $\mathrm{C}+\mathrm{T}$ were at $49-51 \%$.

Note that control regions with genome-average $X$-composition also have genome-averaged nonX-composition. Therefore, their subsitution ratios are in inverse proportion to each other: $S_{X}=1 / S_{\text {nonX }}$. Due to this only one ratio for $X$ and non $X$ pair is shown in Figures 1 and 2. 


\section{Calculation of the substitution ratios in MRI and control regions}

Studying SNPs and fixed substitutions in X-rich MRI regions we measured the number of changes from $X$ to nonX (denoted as $N_{X \rightarrow \text { nonX }}$ ) and also the number of changes from non $X$ to $X$ (denoted as $N_{n o n X \rightarrow X}$ ). The fluctuations in the observed distribution of $N_{X \rightarrow n o n X}$ and $N_{n o n X \rightarrow X}$ are well-known as Poisson noise. Thus, the standard deviation for the true values for $N_{X \rightarrow n o n X}$ and $N_{n o n X \rightarrow X}$ is calculated according to $v$ the Poisson distribution, that is: $\sigma_{N}=$ $\sqrt{N}$. For each $X$-rich MRI region we measured the substitution $S_{X}$-ratios with $S_{X}=N_{X \rightarrow \text { nonX }} / N_{n o n X \rightarrow X}$ shown in Figures 1 and 2 . The propagation of uncertainty for a ratio $f$ $=A / B$ can be calculated using the formula $\left(\sigma_{f} / f\right)^{2}=\left(\sigma_{A} / A\right)^{2}$ $+\left(\sigma_{B} / B\right)^{2}-2\left(\sigma_{A} \cdot \sigma_{B}\right) /(A \cdot B) \cdot \rho_{A B^{\prime}}$ where $\rho_{A B}$ is the correlation coefficient for $A$ and $B$ variables. Because the observed frequency of having a SNP at a genomic site in humans is less than $1 \%$, it is correct to assume that the correlation between $N_{X \rightarrow n o n X}$ and $N_{\text {nonX } \rightarrow X}$ is negligible. Therefore, the standard deviation for the $S_{X}$ ratio was calculated by the following formula:

$$
\sigma=\left(N_{X} / N_{n o n X}\right) \sqrt{\left(1 / N_{X}\right)+\left(1 / N_{n o n X}\right)}
$$

\section{Calculation of base composition equilibrium for the observed substitution rates}

As described in the previous paragraphs, for studying SNPs and fixed substitutions in X-rich MRI regions of the human genome we measured the number of changes from $X$ to non $X$ (denoted as $N_{X \rightarrow \text { non } X}$ ) and also the number of changes from non $X$ to $X$ (denoted as $N_{n o n X \rightarrow X}$ ). These $N_{X \rightarrow \text { non } X}$ and $N_{n o n X \rightarrow X}$ helped us to estimate the frequencies of these two types of mutations per $X$ or nonX site, named here as $F_{X \rightarrow \text { non } X}$ and $F_{n o n X \rightarrow X}$ correspondingly. Suppose one has a sample of MRI regions with a total nucleotide sequence length of $L$ and a composition of $X$ with the region richness given as $P_{X}$ being measured in numbers from 0 to 1 . Then, the total number of $X$ sites in this sample will be $L \cdot P_{X}$, and the total number of nonX sites will be $L \cdot\left(1-P_{X}\right)$. During a certain time interval called $\Delta T$ there will be $\Delta N_{X \rightarrow n o n X}$ and $\Delta N_{n o n X \rightarrow X}$ substitutions. Therefore the frequency of substitutions per site is $F_{X \rightarrow \text { nonX }}=$ $\Delta N_{X \rightarrow \text { non } X} /\left(\Delta T \cdot L \cdot P_{X}\right)$ and $F_{\text {non } X \rightarrow X}=\Delta N_{\text {non } X \rightarrow X} /(\Delta T \cdot L \cdot(1-$ $\left.P_{X}\right)$ ). It is impossible to measure directly these $\Delta N$ values for a specific time interval of $\Delta T$. However, with a good approximation we can assume that the frequencies are proportional to the observed numbers $N_{X \rightarrow n o n X}$ and $N_{\text {non } X \rightarrow X}$ and can be represented by the simple formula: $F_{X \rightarrow \text { non } X}=A \cdot N_{X \rightarrow \text { non } X} / P_{X}$ and $F_{n o n X \rightarrow X}=A \cdot N_{\text {non } X \rightarrow X} /\left(1-P_{X}\right)$, where $A$ is a scaling factor having the same value for $F_{X \rightarrow \text { non } X}$ and $F_{n o n X \rightarrow X^{\prime}}$ since $N_{X \rightarrow \text { nonX }}$ and $N_{\text {nonX } \rightarrow X}$ are counted from the same sample. In a gedanken experiment, let's assume that the current $F_{X \rightarrow \text { nonX }}$ and $F_{n o n X \rightarrow X}$ values will stay unchangeable forever for our MRI sample. Then, in time, mutations should alter the base composition of our sample until it reaches an equilibrium composition with a new percentage for $X$-bases denoted here as $Q_{X}$. This equilibrium composition $Q_{X}$ can be computed using the observed parameters of $P_{X^{\prime}} N_{X \rightarrow \text { non } X^{\prime}}$ and $N_{\text {nonX } \rightarrow X}$. Indeed, under the equilibrium, the number of changes from $X$ to non $X$ must be equal to the number of reverse changes from non $X$ to $X$, or:

$$
\Delta \mathrm{N}_{X \rightarrow n o n X}=\Delta \mathrm{N}_{n o n X \rightarrow X}
$$

We can compute these $\Delta N_{X \rightarrow \text { nonX }}$ and $\Delta N_{n o n X \rightarrow X}$ values from frequencies in such a way:

$\Delta N_{X \rightarrow n o n X}=F_{X \rightarrow n o n X} \cdot \Delta T \cdot L \cdot Q_{X}=A \cdot \frac{N_{X \rightarrow n o n X}}{P_{X}} \cdot \Delta T \cdot L \cdot Q_{X}$

also in a similar way

$\Delta N_{n o n X \rightarrow X}=F_{n o n X \rightarrow X} \cdot \Delta T \cdot L \cdot\left(1-Q_{X}\right)=A \cdot \frac{N_{\text {non } X \rightarrow X}}{1-P_{X}} \cdot \Delta T \cdot L \cdot\left(1-Q_{X}\right)$

By putting these transformations into Equation 1 we get:

$A \cdot \Delta T \cdot L \cdot Q_{X} \cdot \frac{N_{X \rightarrow \text { nonX }}}{P_{X}}=A \cdot \Delta T \cdot L \cdot\left(1-Q_{X}\right) \cdot \frac{N_{n o n X} \rightarrow X}{\left(1-P_{X}\right)}$

or

$$
Q_{X} \cdot \frac{N_{X \rightarrow n o n X}}{P_{X}}=\left(1-Q_{X}\right) \cdot \frac{N_{n o n X \rightarrow X}}{\left(1-P_{X}\right)}
$$

Finally, simple transformation of Equation 2 gives us the final Equation 3 for calculation of equilibrium percentage:

$$
Q_{X}=\frac{P_{X} \cdot N_{n o n X} \rightarrow X}{N_{X} \cdot\left(1-P_{X}\right)+N_{n o n X} \rightarrow X \cdot P_{X}}
$$

In the Results section, Formula 3 is used to compute the equilibrium percentage for $X$-bases in the studied MRI regions.

\section{Abbreviations}

MRI: mid-range inhomogeneity; SNP: Single nucleotide polymorphisms; Mb: Megabase(s); indels: insertions and deletions; nt: nucleotide(s).

\section{Authors' contributions}

$\mathrm{AP}, \mathrm{BRH}, \mathrm{SPA}, \mathrm{MC}$, JH, OMB were responsible for computational processing of the human-chimp-macaque datasets and creating the described programs. SS was responsible for the procuring and processing of the SNP 
data from dbSNP. JMB was responsible for the quantification of SNP data in the alignment. AP was also responsible for the processing of fixed substitutions and indel data from the three way alignment. AF supervised the project, provided guidance and wrote the draft. SS and AP also contributed to editing, typesetting, and writing the draft. All authors have read and approved the final manuscript.

\section{Additional material}

\section{Additional file 1}

S1 - SNP dataset. Complete set of data representing the number of occurrences of major, middle, minor and rare SNPs in all X MRI regions (where $\mathrm{X}$ could represent either a two-base combination [GC, AG, GT, etc.] or a single base) and their corresponding control regions, which have an average nucleotide composition for the respective $\mathrm{X}$.

Click here for file

[http://www.biomedcentral.com/content/supplementary/14712164-10-513-S1.xls]

\section{Additional file 2}

$S 2 A$ - Indel data for combination of nts, S2B - Indel data for individual nts. $\bullet 2 A$ - Complete set of data representing the total number of $\mathrm{X}$ nucleotides (where $\mathrm{X}$ represents a two base combination such as $G C, A G$, $G T$, etc.) inserted or deleted due to short indels, whose sizes are less than three nucleotides in length, or medium indels, whose sizes range from three to fifty nucleotides in length, for all X MRI regions, and for the control regions, which have an average nucleotide composition for the respective $\mathrm{X} .-2 B$ - Complete set of data representing the total number of $\mathrm{X}$ nucleotides (where $\mathrm{X}$ represents a single base) inserted or deleted due to short indels, whose sizes are less than three nucleotides in length, or medium indels, whose sizes range from three to fifty nucleotides in length, for all X MRI regions, and for the control regions, which have an average nucleotide composition for the respective $\mathrm{X}$.

Click here for file

[http://www.biomedcentral.com/content/supplementary/14712164-10-513-S2.xls]

\section{Additional file 3}

S3 - Dataset of fixed substitutions. Complete set of data representing the number of occurrences of fixed substitutions on all X MRI regions (where $\mathrm{X}$ could represent either a two-base combination [GC, AG, GT, etc.] or a single base), and the control regions, which have an average nucleotide composition for the respective $\mathrm{X}$.

Click here for file

[http://www.biomedcentral.com/content/supplementary/14712164-10-513-S3.xls]

\section{Acknowledgements}

This project is supported by NSF Career award MCB-0643542. We thank Peter Bazeley, University of Toledo, for his computational support and discussion of our algorithms.

\section{References}

I. Consortium IHG: Finishing the euchromatic sequence of the human genome. Nature 2004, 43 I (70 I I):93I-945.

2. Suzuki M, Hayashizaki Y: Mouse-centric comparative transcriptomics of protein coding and non-coding RNAs. Bioessays 2004, 26(8):833-843.
3. Segal E, Fondufe-Mittendorf $Y$, Chen L, Thastrom A, Field $Y$, Moore IK, Wang JPZ, Widom J: A genomic code for nucleosome positioning. Nature 2006, 442(7104):772-778.

4. Chattopadhyay S, Pavithra L: MARs and MARBPs: key modulators of gene regulation and disease manifestation. Subcell Biochem 2007, 4I:2 13-230.

5. Karlin S, Burge C: Dinucleotide relative abundance extremes: a genomic signature. Trends Genet 1995, I I (7):283-290.

6. Csuros M, Noe L, Kucherov G: Reconsidering the significance of genomic word frequencies. Trends Genet 2007, 23(I I):543-546.

7. Rigoutsos I, Huynh T, Miranda K, Tsirigos A, McHardy A, Platt D: Short blocks from the noncoding parts of the human genome have instances within nearly all known genes and relate to biological processes. Proc Natl Acad Sci USA 2006, 103(I7):6605-66I0.

8. Meynert A, Birney E: Picking pyknons out of the human genome. Cell 2006, I 25(5):836-838.

9. Bernardi G: The vertebrate genome: isochores and evolution. Mol Biol Evol 1993, 10:186-204.

10. Karlin S, Campbell AM, Mrazek J: Comparative DNA analysis across diverse genomes. Annu Rev Genet 1998, 32:185-225.

II. Bechtel JM, Wittenschlaeger T, Dwyer T, Song J, Arunachalam S, Ramakrishnan SK, Shepard S, Fedorov A: Genomic mid-range inhomogeneity correlates with an abundance of RNA secondary structures. BMC Genomics 2008, 9:284.

12. Mrazek J, Kypr J: Middle-range clustering of nucleotides in genomes. Comput Appl Biosci 1995, I I(2): 195-199.

13. Nikolaou C, Almirantis $Y$ : $\mathbf{A}$ study of the middle-scale nucleotide clustering in DNA sequences of various origin and functionality, by means of a method based on a modified standard deviation. J Theor Biol 2002, 21 7(4):479-492.

14. Elson $D$, Chargaff $E$ : On the desoxyribonucleic acid content of sea urchin gametes. Experientia I952, 8(4): I43-5.

15. Kimura M: The Neutral theory of molecular evolution New York: Cambridge University Press; 1983.

16. Gardiner-Garden M, Frommer M: CpG islands in vertebrate genomes. J Mol Biol 1987, 196(2):26I-282.

17. Takai D, Jones PA: The CpG island searcher: a new WWW resource. In Silico Biol 2003, 3(3):235-240.

18. Webster MT, Smith NGC: Fixation biases affecting human SNPs. Trends Genet 2004, 20(3): I22-126.

19. Duret L, Eyre-Walker A, Galtier N: A new perspective on isochore evolution. Gene 2006, 385:71-74.

20. Sequencing $C$, Consortium A: Initial sequence of the chimpanzee genome and comparison with the human genome. Nature 2005, 437(7055):69-87.

21. Kuhn R, Karolchik D, Zweig A, Wang T, Smith K, Rosenbloom K, Rhead B, Raney B, Pohl A, Pheasant M, Meyer L, Hsu F, Hinrichs A, Harte R, Giardine B, Fujita P, Diekhans M, Dreszer T, Clawson H, Barber G, Haussler D, Kent W: The UCSC Genome Browser Database: update 2009. Nucleic Acids Res 2008:D775-6I.

22. Sherry ST, Ward MH, Kholodov M, Baker J, Phan L, Smigielski EM, Sirotkin K: dbSNP: the NCBI database of genetic variation. Nucleic Acids Res 200 I, 29:308-3I I.

Publish with Bio Med Central and every scientist can read your work free of charge

"BioMed Central will be the most significant development for disseminating the results of biomedical research in our lifetime. "

Sir Paul Nurse, Cancer Research UK

Your research papers will be:

- available free of charge to the entire biomedical community

- peer reviewed and published immediately upon acceptance

- cited in PubMed and archived on PubMed Central

- yours - you keep the copyright 